\title{
Fast-Twitch Muscle Fiber
}

National Cancer Institute

\section{Source}

National Cancer Institute. Fast-Twitch Muscle Fiber. NCI Thesaurus. Code C12613.

A large-diameter skeletal muscle fiber that uses glycogen reserves rather than oxygenrich blood for quick energy and a more rapid production of force. Fast twitch muscle fibers have a low oxidative capacity and can produce ATP without the need for oxygen, resulting in a higher activation threshold and a higher velocity signal conduction. 